\title{
La base de données Medicus Quebecensis sur internet : une fenêtre sur les articles de revues médicales québécoises au XIX siècle
}

\section{François Guérard}

Volume 53, numéro 1, été 1999

Médecine, santé et sociétés

URI : https://id.erudit.org/iderudit/005460ar

DOI : https://doi.org/10.7202/005460ar

Aller au sommaire du numéro

Éditeur(s)

Institut d'histoire de l'Amérique française

ISSN

0035-2357 (imprimé)

1492-1383 (numérique)

Découvrir la revue

Citer cet article

Guérard, F. (1999). La base de données Medicus Quebecensis sur internet : une fenêtre sur les articles de revues médicales québécoises au XIX ${ }^{\mathrm{e}}$ siècle. Revue d'histoire de l'Amérique française, 53(1), 121-122.

https://doi.org/10.7202/005460ar d'utilisation que vous pouvez consulter en ligne. 


\title{
LA BASE DE DONNÉES MEDICUS QUEBECENSIS SUR INTERNET : UNE FENÊTRE SUR LES ARTICLES DE REVUES MÉDICALES QUÉBÉCOISES AU XIX' ${ }^{\mathrm{e}}$ SIÈCLE
}

\author{
FRANÇOIS GUÉRARD \\ Centre interuniversitaire d'études québécoises \\ Université du Québec à Trois-Rivières
}

Un nouvel outil destiné aux chercheurs en histoire de la santé et de la médecine au Québec vient de voir le jour sur Internet : MEDICUS QUEBECENSIS, une base de données qui recense pas moins de 6882 articles de revues médicales québécoises parus au $\mathrm{XIX}^{\mathrm{e}}$ siècle et dont les auteurs ont résidé au Québec. Logée sur le site INTERNET du Centre interuniversitaire d'études québécoises, la base de données peut être interrogée simultanément par plusieurs chercheurs selon divers critères de recherche.

Les origines de cet outil de recherches remontent à plusieurs années, alors que feu André Paradis et ses assistants ont systématiquement dépouillé une vingtaine de revues médicales dont ils ont extrait des milliers de références. Ils avaient comme objectif d'en publier un index par auteur, par spécialité médicale, par maladie et par médicament. Le décès de monsieur Paradis paraissait avoir compromis le projet. Mais sa documentation a été confiée à François Guérard, spécialiste de l'histoire de la santé au Québec qui, avec l'accord de la veuve d'André Paradis, a pu compléter l'instrument de recherche.

L'implantation de MEDICUS QUEBECENSIS sur un site Internet comporte plusieurs avantages. La base de données peut être consultée en tous temps, sans frais, par des chercheurs dispersés sur le territoire québécois ou hors du Québec. Les usagers peuvent aisément - et ils y sont d'ailleurs invités — faire part de leurs commentaires, suggérer des modifications ou même des corrections. Ainsi, la présentation et le contenu, sont appelés à évoluer selon les remarques des usagers, faisant de la base de données un outil interactif.

Pour le Centre interuniversitaire d'études québécoises qui rend accessible cette base de données sur Internet, il s'agit d'un projet-pilote. En effet, le CIEQ compte emprunter la voie des réseaux informatiques pour offrir à la communauté scientifique d'autres ensembles de données issues des tra- 
vaux de ses chercheurs. Ces nouveaux projets partageront avec MEDICUS QUEBECENSIS une même philosophie : miser prioritairement sur le contenu et le mettre en valeur à l'aide de moyens somme toute modestes, dans une présentation par conséquent assez sobre. Chaque document sera accompagné de notes explicatives qui en préciseront la portée et les limites.

\section{PÉRIODIQUES DÉPOUILLÉS ET NOMBRE D'ARTICLES RECENSÉS}

\begin{tabular}{|c|c|c|}
\hline Périodique & Articles & Période \\
\hline L'Abeille médicale & 88 & $1879-1882$ \\
\hline The British American Journal & 68 & $1860-1862$ \\
\hline The British American Journal of Medical and & & \\
\hline Physical Science & 141 & $1845-1850$ \\
\hline The British American Medical and Physical & & \\
\hline Journal & 40 & $1850-1851$ \\
\hline Le Bulletin médical de Québec & 37 & 1899 \\
\hline Canada Lancet & 41 & $1863-1864$ \\
\hline Canada Medical and Surgical Journal & 1416 & $1872-1888$ \\
\hline $\begin{array}{l}\text { Canada Medical Journal and Monthly Record of } \\
\text { Medical and Surgical Science (1) }\end{array}$ & 60 & $1852-1853$ \\
\hline $\begin{array}{l}\text { Canada Medical Journal and Monthly Record of } \\
\text { Medical and Surgical Science (2) }\end{array}$ & 283 & $1864-1872$ \\
\hline The Canada Medical Record & 1292 & 1872-1899 \\
\hline La Clinique & 143 & 1894-1899 \\
\hline La Gazette médicale & 24 & $1865-1866$ \\
\hline La Gazette médicale de Montréal & 188 & $1887-1892$ \\
\hline Journal d'hygiène populaire & 508 & $1884-1895$ \\
\hline Journal de médecine de Québec & 42 & $1826-1827$ \\
\hline La Lancette canadienne & 13 & 1847 \\
\hline The Medical Chronicle or Montreal Monthly & & \\
\hline Journal of Medecine and Surgery & 163 & 1853-1859 \\
\hline The Montreal Medical Gazette & 63 & $1844-1845$ \\
\hline The Montreal Medical Journal & 950 & 1888-1899 \\
\hline La Revue médicale & 191 & 1897-1899 \\
\hline L'Union médicale du Canada & 1131 & $1872-1899$ \\
\hline Nombre total d'articles & 6882 & \\
\hline
\end{tabular}

Pour accéder à MEDICUS QUEBECENSIS : se rendre à l'adresse : http://www.cieq.ulaval.ca/ sélectionner «Accès en ligne aux bases de données du CIEQ » sélectionner « MEDICUS QUEBECENSIS » 